Hambley, T. W. (1987). MOMEC87. Program for strain-energy minimization. Univ. of Sydney, Australia.

Hambley, T. W., Gahan, L. R. \& Searle, G. H. (1988). Acta Cryst. Submitted.

Hambley, T. W., Hawkins, C. J., Palmer, J. A. \& Snow, M. R. (1981). Aust.J. Chem. 35, 34-45.

Hambley, T. W. \& Snow, M. R. (1986). Inorg. Chem. 25, 1378-1382.

Hammershoi, A., Larsen, E. \& Larsen, S. (1978). Acta Chem. Scand. Ser. A, 32, 501-507.

Hidaka, J., Yamada, S. \& Shimura, Y. (1974). Chem. Lett. pp. 1487-1490.
International Tables for X-ray Crystallography (1974). Vol. IV. Birmingham: Kynoch Press. (Present distributor Kluwer Academic Publishers, Dordrecht.)

JoHNSON, C. K. (1965). ORTEP. Report ORNL-3794. Oak Ridge National Laboratory, Tennessee, USA.

Karplus, M. \& MCCAmmon, J. A. (1981). CRC Crit. Rev. Biochem. 9, 293.

Searle, G. H. \& Larsen, E. (1976). Acta Chem. Scand. Ser. A, 30, 143-151.

SHELDRICK, G. M. (1976). SHELX76. Program for crystal structure determination. Univ. of Cambridge, England.

Tulip, W. R., COOPER, M. K., Hambley, T. W., Murdoch, C. M. \& FREEMAN, H. C. (1988). In preparation.

Acta Cryst. (1988). B44, 609-616

\title{
The Influence of Intermolecular Interactions on the Electron-Density Distribution. A Comparison of Experimental and Theoretical Results for $\alpha$-Oxalic Acid Dihydrate*
}

\author{
By M. P. C. M. KriJn, H. GraAfsma † and D. FeIL \\ Chemical Physics Laboratory, University of Twente, PO Box 217, 7500 AE Enschede, The Netherlands
}

(Received 10 March 1988; accepted 16 May 1988)

\begin{abstract}
The experimental electron-density distribution in $\alpha$ oxalic acid dihydrate, $\alpha-\mathrm{C}_{2} \mathrm{H}_{2} \mathrm{O}_{4} \cdot 2 \mathrm{H}_{2} \mathrm{O}$, as measured by Dam, Harkema \& Feil [Acta Cryst. (1983), B39, $760-768]$, is compared with results from a theoretical density-functional calculation, with a local approximation to exchange and correlation. The agreement between the multipole-refined experimental and the refined vibrationally averaged theoretical electrondensity distribution improves significantly when taking into account the effects of hydrogen bonding and crystal environment. A comparison of structure factors based on the experimental electron-density distribution with those based on the vibrationally averaged theoretical molecular-density distributions, yielded an $R$ factor of $1.3 \%$. Inclusion of the effects of hydrogen bonding in the theoretical model lowered the $R$ factor to $1 \cdot 1 \%$. When the effects of the crystal environment were taken into account, a further lowering to $1.0 \%$ resulted.
\end{abstract}

\section{Introduction}

As part of a recent project of the International Union of Crystallography, the electron-density distribution in $\alpha$-oxalic acid dihydrate has been thoroughly investigated, using both experimental and theoretical

\footnotetext{
* Supported in part by the Netherlands Foundation for Chemical Research (SON) with financial aid from the Netherlands Organization for Advancement of Pure Research (ZWO).

+ Present address: Chemistry Department, State University of New York at Buffalo, Buffalo, New York 14214, USA.
}

0108-7681/88/060609-08\$03.00 techniques (Coppens et al., 1984). The largest discrepancies between experiment and theory appeared to occur in the lone-pair regions where theoretical deformation densities (i.e. the difference between the molecular density and the superimposed spherically averaged densities of the free atoms constituting the molecule) are higher and less diffuse compared with the experimental deformation density. The cause of this discrepancy may well be ascribed to the limited basis set used and, as suggested by Olovsson (1980), Stevens (1980) and Hermansson $(1984,1985)$, the neglect of taking into account intermolecular interactions in the theoretical calculations.

The main object of this study is a comparison of the electron-density distribution in $\alpha$-oxalic acid dihydrate obtained from $\mathrm{X}$-ray diffraction experiments on single crystals with those obtained from quantum-mechanical calculations. Therefore, the subject of a theoretical calculation should also be a system consisting of a large cluster of properly oriented and positioned interacting molecules. Since this approach is not feasible from a computational point of view, the model has to be simplified considerably.

Previous studies (Krijn \& Feil, 1986, 1987) indicated that the electron-density redistribution, upon forming weak hydrogen bonds, is dominated by a polarization contribution. Consequently, the effects of weak hydrogen bonds on the electron-density distribution can be accounted for by placing the molecule in the electric field of its weakly bonded neighbours. In the case of strong hydrogen bonds, however, the subtle interplay of exchange repulsion, charge transfer and polarization

c) 1988 International Union of Crystallography 
requires a quantum-mechanical description of the complex or supermolecule consisting of the strongly bonded molecules. The elementary building block of crystalline $\alpha$-oxalic acid dihydrate is the oxalic acid dihydrate complex, bonded to neighbouring complexes by hydrogen bonds of weak and intermediate strength. Therefore, we focus on one such complex, explicitly taking into account its internal strong hydrogen bonds, and approximate the influence of the crystal environment by including its electrostatic potential in the Hamiltonian describing the complex.

From $a b$ initio SCF Hartree-Fock (HF) calculations on the oxalic acid dihydrate complex (Breitenstein et al., 1983), employing a 4-31G basis set augmented with bond-centred polarization functions, it was concluded that taking into account the contribution of the internal hydrogen bonds to the electron-density distribution does not markedly improve the discrepancy between experiment (Stevens \& Coppens, 1980) and theory. However, based on a similar study (Krijn \& Feil, 1988), we predicted just the contrary, namely a measurable increase, at least in principle, of electron density in the $\mathrm{O}-\mathrm{H}$-bond region of the oxalic acid molecule, improving the agreement between experiment (Dam, Harkema \& Feil, 1983) and theory significantly. The change in oxygen lone-pair density of the water molecule, induced by the hydrogen bond donated by the oxalic acid molecule, was found not to exceed the experimental standard deviation.

To calculate the electron-density distribution, the formalism of density-functional theory was adopted, with a local approximation to exchange and correlation, which proved to be a viable and computationally less-demanding alternative to the HF formalism, without the expense of accuracy. The method allows the use of a mathematically nearly complete basis set for systems as large as oxalic acid dihydrate. The basis-set dependence of the electron-density distribution has been investigated by Bicerano, Marynick \& Lipscomb (1978), Breitenstein et al. (1983), and Baerends et al. (1985), from which we infer that inclusion of $d$ - and $f$-type polarization functions is a prerequisite for obtaining reliable electron-density distributions, i.e. density distributions with an accuracy better than $0.1 \mathrm{e} \AA^{-3}$ in the bonding regions.

A direct comparison of experiment and theory is hampered on account of the first being a time average over the internal and lattice vibrations. The aim of the present paper is to tackle this problem by performing a multipole refinement of both the experimental and dynamic (i.e. vibrationally averaged) theoretical results and afterwards matching the parameters describing the temperature dependence of the electron-density distribution. In this manner, experiment and theory are treated on a equal footing, eliminating for the greater part the bias introduced by refinement model inadequacies, while spurious features in the experimental density distribution are filtered out. Possible inadequacies of the refinement model are investigated and discussed.

\section{Methodology}

\section{Experiment}

Results of the determination of the electron-density distribution in $\alpha$-oxalic acid dihydrate at $100 \mathrm{~K}$, by means of X-ray diffraction, were reported by Dam et al. (1983). An extensive data set, consisting of all reflections in the reciprocal sphere up to $(\sin \theta) / \lambda$ $=1 \cdot 3 \AA^{-1}$, was measured. Structural parameters and deformation densities compared well, whereas thermal parameters differed significantly from the findings of an earlier published study (Stevens \& Coppens, 1980). Correction for thermal diffuse scattering increased the vibrational parameters by $12 \%$, without however, resulting in significant deviations in the deformation density. A perturbation of the water oxygen lone-pair density was perceived and attributed to hydrogen bonding of the water molecule to oxalic acid.

\section{Quantum-mechanical calculations}

Quantum-mechanical calculations of the electrondensity distribution in the oxalic acid dihydrate complex were performed within the framework of densityfunctional theory. The local approximation to the exchange-correlation potential employed was the exchange potential of Kohn \& Sham (1965) added to the correlation potential represented by the parametrization by Vosko \& Wilk (1980) and Vosko, Wilk \& Nusair (1980) of the Monte Carlo results of Ceperley \& Alder (1980) for the homogeneous electron gas. The correlation part of the exchange-correlation potential was corrected for self-interaction according to the method of Stoll, Pavlidou \& Preuss (1978) and Stoll, Golka \& Preuss (1980). Matrix elements in the secular equation were determined numerically (Baerends \& Ros, 1978). The basis set consisted of Cartesian Slater-type orbital (STO) functions with polarization functions up to $l=2$ on $\mathrm{H}$ and up to $l=3$ on $\mathrm{C}$ and $\mathrm{O}$. Results were corrected for the basis-set superposition error according to the counterpoise method of Boys \& Bernardi (1970).

The geometry of the oxalic acid dihydrate complex was obtained from bond distances and angles determined by neutron diffraction at $100 \mathrm{~K}$ (Koetzle \& McMullan, 1980).

Computational details and results are reported elsewhere (Krijn \& Feil, 1988).

Since the analytical Fourier transform of a twocentre product of STO's is not feasible, the theoretical electron-density distribution $\rho$ was expanded in a finite 
set of atom-centred spherical harmonics, multiplied by a Slater-type radial part,

$$
\rho \simeq \tilde{\rho}=\sum_{i} a_{i} f_{i} .
$$

The expansion coefficients $a_{i}$ were computed from an analytical minimization of the error function

$$
D=\int(\rho-\tilde{\rho})^{2} \mathrm{~d}^{3} r
$$

subject to the norm preserving constraint

$$
\int \tilde{\rho} \mathrm{d}^{3} r=N_{e},
$$

where $N_{e}$ is the total number of electrons (Baerends \& Ros, 1978). The functions $f_{i}$, extending up to $l=4$ on each atomic site, were chosen in such a manner as to result in an error $|\rho-\tilde{\rho}|$ less than $0.025 e \AA^{-3}$ everywhere. Structure factors were calculated analytically from $\tilde{\rho}$ (Kaijser \& Smith, 1977).

\section{Multipole refinement}

The experimental and dynamic theoretical electrondensity distributions are refined, retaining the convolution approximation (Coulson \& Thomas, 1971; Stewart, 1976). The electron-density distribution is divided into atomic fragments that are assumed to undergo rigid harmonic translational vibrations only. The density $\rho_{i}$ of atomic fragment $i$ is expressed as a superposition of the spherically averaged free-atom density $\rho_{i}^{\text {at }}$, plus a deformation density expressed as a linear combination of deformation functions, identical to a sum of spherical harmonics with a Slater-type radial part, centred at the site of atom $i$,

$\rho_{i}(\mathbf{r})=\rho_{i}^{\mathrm{at}}(\mathbf{r})+\sum_{n} \sum_{k} C_{i, n, k} N_{n} r_{i}^{n} \exp \left(-\alpha_{i} r_{i}\right) \cos ^{n} \theta_{i, k}$,

in which $r_{i}$ is the distance from the atomic centre and $\theta_{i, k}$ the angle between the radius vector $\mathbf{r}_{i}$ and $\mathbf{a}$ specified axis $\mathbf{k}$ through the atomic centre (Hirshfeld, 1971, 1977). $\alpha_{i}$ is an adjustable shape parameter for each atom $i, n$ an integer between 0 and 4 , and $N_{n}$ a normalization factor depending on $n$ and $\alpha$.

For all non- $\mathrm{H}$ atoms, the coefficients $C_{i, n, k}$ and parameters $\alpha_{i}$ are refined together with the positional and thermal parameters, whereas for $\mathrm{H}$ atoms only $C_{i, n, k}$ and $\alpha_{i}$ are refined. Functions with $n=3$ and $n=4$ were omitted for all $\mathrm{H}$ atoms. No symmetry constraints, other than the crystallographic symmetry, are imposed, resulting in 7 exponential parameters $\alpha_{i}(3$ for $\mathrm{H}, 1$ for $\mathrm{C}$, and 3 for $\mathrm{O}$ ) and 169 expansion coefficients $C_{i, n, k}(30$ for $\mathrm{H}, 35$ for $\mathrm{C}, 105$ for $\mathrm{O}$, minus 1 neutrality constraint). Including a scale factor, 12 positional and 24 thermal parameters, this amounts to 213 independent parameters.
Table 1. Experimental atomic coordinates $\left(\times 10^{5}\right)$ and thermal parameters $\left(\AA^{2} \times 10^{5}\right)$ for non- $\mathrm{H}$ atoms, resulting from a high-order refinement $[\mathrm{HO}$, first row, taken from Dam, Harkema \& Feil (1983)], multipole refinement (MR 1 , second row) and multipole refinement with theoretically derived scale factor (MR2, third row)

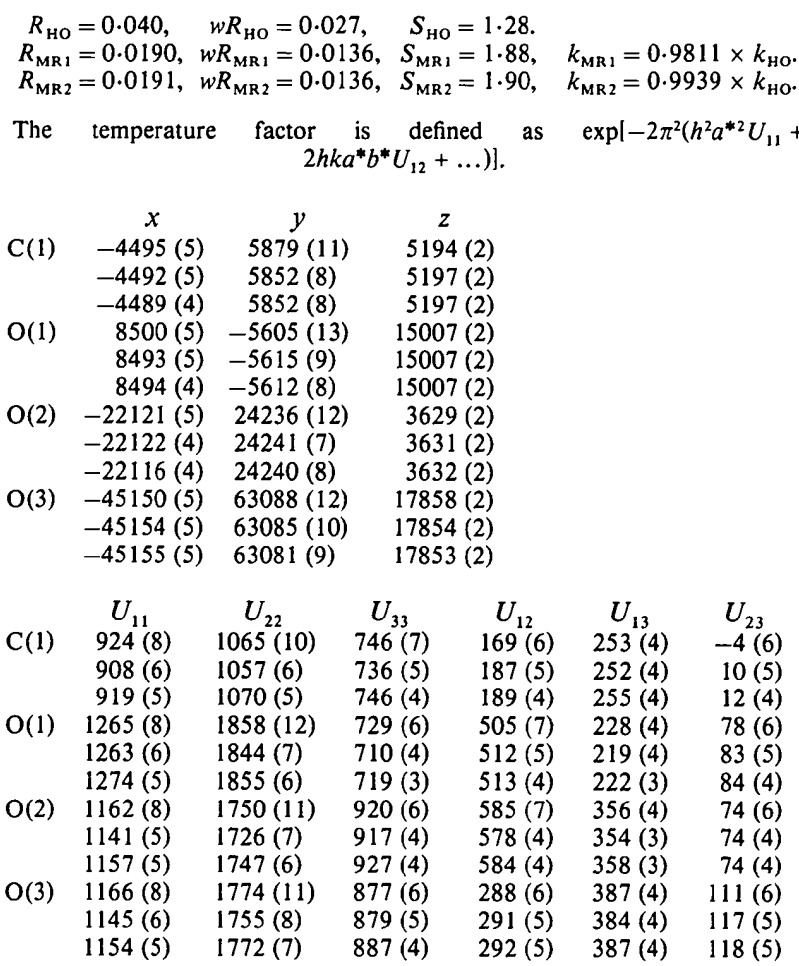

\section{Results and discussion}

\section{Multipole refinement of experiment}

A full-angle refinement was performed, using the cell parameters and X-ray data of Dam et al. (1983), corrected for extinction and anomalous dispersion. Positional and thermal parameters of the $\mathrm{H}$ atoms were kept fixed at the values taken from the neutron diffraction study of Koetzle \& McMullan (1980). The converged refinement resulted in the discrepancy indices

$$
\begin{aligned}
R & =\sum\left|F_{o} \cdots k\right| F_{c}|| / \sum\left|F_{o}\right|=0.0190 \\
w R & =\left[\sum w\left(F_{o}-k F_{c}\right)^{2} / \sum w F_{o}^{2}\right]^{1 / 2}=0.0136 \\
S & =\left[\sum w\left(F_{o}-k F_{c}\right)^{2} /(N-p)\right]^{1 / 2}=1.88,
\end{aligned}
$$

where $w=1 / \sigma^{2}(F) . \sigma(F)$ was calculated from $\sigma(I)$ according to Hamilton (1964). $N$ and $p$ are the number of refined structure factors (3304) and refined parameters (213), respectively. The positional and thermal parameters of the high-order [labelled HO and taken from Dam et al. (1983)] and multipole refinement (labelled MR 1) are compared in Table 1, and seen to be in good agreement. Asphericity shifts and the average 
values of positional parameter discrepancies with the neutron diffraction results of Koetzle \& McMullan (1980) do not exceed $0.001 \AA$. Although the deviations between X-ray and neutron diffraction thermal parameters exceed statistical criteria, the agreement is remarkable (see also Coppens et al., 1984).

The multipole-refined dynamic deformation density is depicted in Figs. $1(a),(b)$, along with the corresponding dynamic residual density in Figs. 2(a), (b). Although not imposed on the multipole model, the carbonyl group remains nearly axially symmetric, as expected.

There is evidence (Stevens \& Coppens, 1980; Swaminathan, Craven, Spackman \& Stewart, 1984) that only a single exponential radial dependence per atom is too restricted a model to describe the density in great detail. Increased flexibility, however, automatically implies an increased correlation between, on the one hand, deformation parameters and, on the other hand, position and thermal parameters. From Figs. $2(a),(b)$, it cannot be concluded whether the model suffices in the present case.

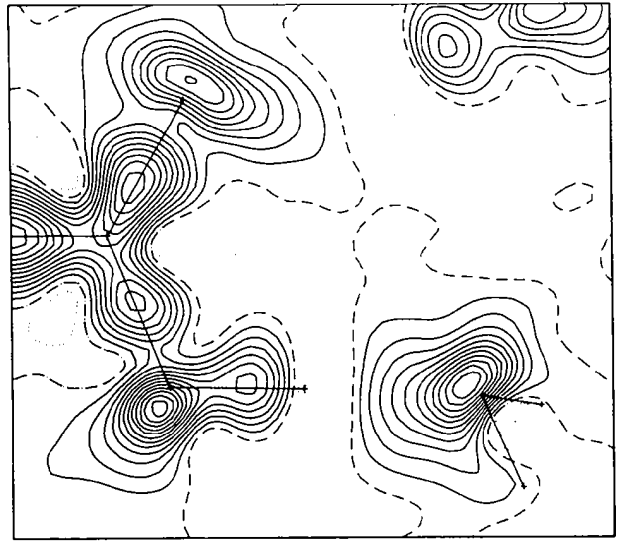

(a)

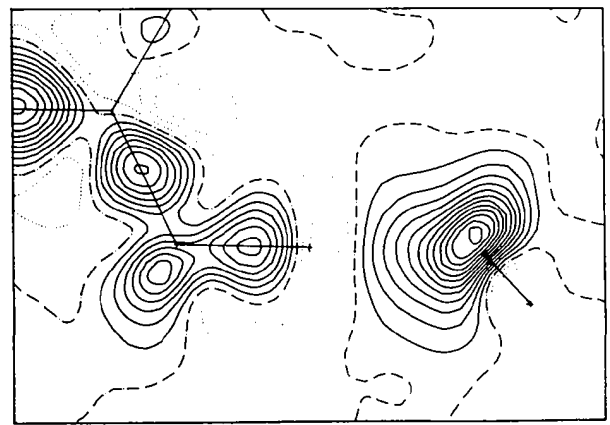

(b)

Fig. 1. Refined experimental dynamic deformation density in oxalic acid dihydrate. Positive contours (electron excess) are drawn as solid lines, zero contours are dash-dotted, and negative contours (electron deficiency) are dotted. The contour interval is $0.05 \mathrm{e}^{-3}\left(1 \mathrm{e} \AA^{-3} \simeq 0.148 \mathrm{e} a_{0}^{-3}\right)$. (a) Deformation density in the plane of the oxalic acid molecule. $(b)$ Deformation density in the plane perpendicular to the water molecule and bisecting the $\mathrm{H}(2)-\mathrm{O}(3)-\mathrm{H}(3)$ angle.

\section{Multipole refinement of theory}

With the object to confirm the adequacy of the refinement model, and to be able to compare experiment and theory on an equal footing, a multipole refinement of the dynamic theoretical density distribution in the oxalic acid dihydrate complex was performed. Structure factors for each scattering vector, corresponding to an experimental measurement, were computed analytically from an expansion of the static theoretical density in spherical harmonics multiplied by a Slater-type radial part [cf. (1)]. Thermal motion was included by applying an overall anisotropic temperature factor (Table 2), obtained from the average of the individual experimental atomic temperature factors, weighted by the number of electrons of the corresponding atoms. In a subsequent refinement with unit weights, positional and thermal parameters of the $\mathrm{H}$ atoms were kept fixed at the input values. The converged refinement resulted in the surprisingly low discrepancy indices $R=0.0023, w R=0.0022$ and $S=0.012$. The input and refined parameters are compared in Table 2. The thermal parameters have undergone a slight increase, contrary to the scale factor. Indeed, correlations

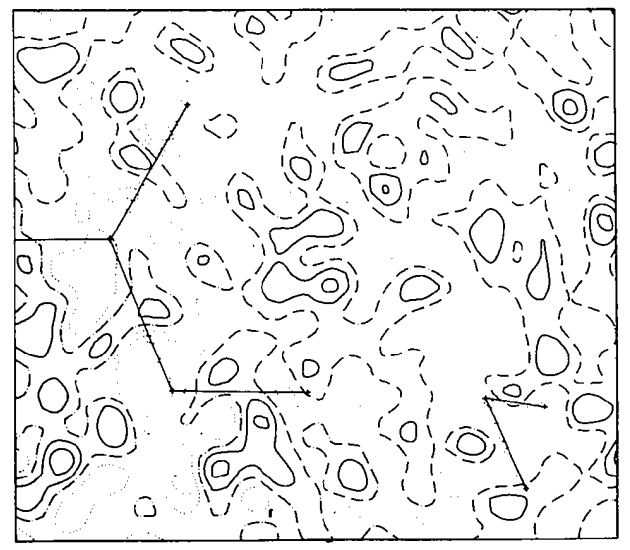

(a)

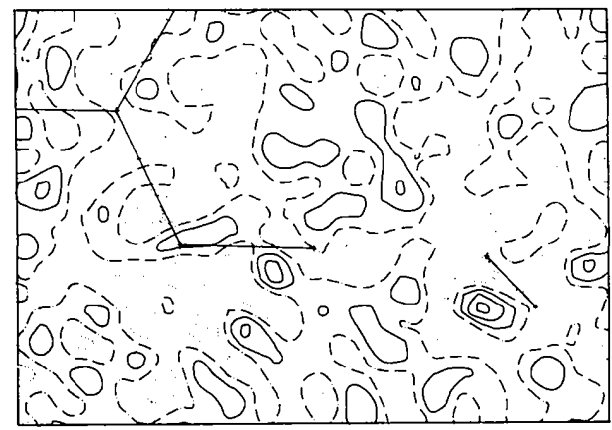

(b)

Fig. 2. Experimental dynamic residual density in oxalic acid

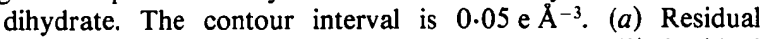
density in the plane of the oxalic acid molecule. (b) Residual density in the plane perpendicular to the water molecule and bisecting the $\mathrm{H}(2)-\mathrm{O}(3)-\mathrm{H}(3)$ angle. 
Table 2. Atomic coordinates $\left(\times 10^{5}\right)$ and thermal parameters $\left(\AA^{2} \times 10^{5}\right)$ for non- $\mathrm{H}$ atoms, resulting from a multipole refinement of the theoretical density of oxalic acid dihydrate

$R=0.0023, \quad w R=0.0022, \quad S=0.012, \quad k=0.9966$ (3).

The temperature factor is defined as $\exp \left[-2 \pi^{2}\left(h^{2} a^{* 2} U_{11}+\right.\right.$ $\left.\left.2 h k a^{*} b^{*} U_{12}+\ldots\right)\right]$.

Input parameters

$\begin{array}{lrrr} & x & y & z \\ \mathrm{C}(1) & -4500 & 5884 & 5201 \\ \mathrm{O}(1) & 8501 & -5589 & 15010 \\ \mathrm{O}(2) & -22128 & 24248 & 3629 \\ \mathrm{O}(3) & -4848 & 13130 & 32139\end{array}$

$\begin{array}{llllllr} & U_{11} & U_{22} & U_{33} & U_{12} & U_{13} & U_{23} \\ \mathrm{C}(1) & 1310 & 1740 & 920 & 420 & 350 & 70 \\ \mathrm{O}(1) & 1310 & 1740 & 920 & 420 & 350 & 70 \\ \mathrm{O}(2) & 1310 & 1740 & 920 & 420 & 350 & 70 \\ \mathrm{O}(3) & 1310 & 1740 & 920 & 420 & 350 & 70\end{array}$

Refined parameters

\begin{tabular}{rrrr} 
& $x$ & \multicolumn{1}{c}{$y$} & \multicolumn{1}{c}{$z$} \\
$\mathrm{C}(1)$ & $-4498(1)$ & $5883(2)$ & $5204(1)$ \\
$\mathrm{O}(1)$ & $8493(1)$ & $-5585(2)$ & $15007(1)$ \\
$\mathrm{O}(2)$ & $-22118(1)$ & $24239(2)$ & $3630(1)$ \\
$\mathrm{O}(3)$ & $-4848(1)$ & $13126(2)$ & $32144(1)$
\end{tabular}

$\begin{array}{ccccccc} & U_{11} & U_{22} & U_{33} & U_{12} & U_{13} & U_{23} \\ \mathrm{C}(1) & 1332(1) & 1768(1) & 945(1) & 423(1) & 354(1) & 73(1) \\ \mathrm{O}(1) & 1326(1) & 1759(1) & 917(1) & 422(1) & 347(1) & 73(1) \\ \mathrm{O}(2) & 1313(1) & 1749(1) & 945(1) & 424(1) & 352(1) & 71(1) \\ \mathrm{O}(3) & 1322(1) & 1759(1) & 930(1) & 403(1) & 357(1) & 70(1)\end{array}$

between thermal and deformation parameters, mediated through their mutual interaction with the scale factor, might be the cause. Especially the cusp functions $(n=0)$ appeared to correlate appreciably $(80 \%)$ with the scale factor. The dynamic residual density (not shown) appeared featureless and nowhere to exceed $0.010 \mathrm{e} \AA^{-3}$, except for peaks with a height of $0.025 \mathrm{e}^{-3}$ centred at the oxalic acid $\mathrm{O}-\mathrm{H}$-bond mid-points. No systematic features are observed in the oxygen lone-pair moieties. The net effect of the small shifts in positional and thermal parameters on the deformation density is depicted in Figs. 3(a), $(b)$. Shown is the difference between the dynamic deformation density corresponding to the refined parameters and the one corresponding to the input parameters. This difference indicates to what extent the model parameters reproduce the deformation density. We expect similar refinement-model errors to be present in the refined experimental deformation density.

\section{Comparison of experiment and theory}

A direct comparison of experiment and theory requires properly accounting for the effect of thermal motion. The latter may be done by either thermally averaging the theoretical density or by deconvolution of the thermal motion from the experimental density. Since no experimental data are available beyond the limiting sphere given by $(\sin \theta) / \lambda=1.3 \AA^{-1}$, the finer details in the static experimental density are unreliable. The dynamic experimental density suffers much less from this deficiency. To compare the dynamic experimental and dynamic theoretical density, the thermal parameters of the refined thermally averaged theoretical density were replaced by the thermal parameters of the refined experimental density. The same matching of parameters was carried out for the slightly differing position parameters.

The theoretically determined scale factor, known on an absolute scale, makes it possible to obtain also an absolute, rather than relative, experimental scale factor. To obtain the latter, we proceeded as follows. After matching the refined theoretical temperature and position parameters to the refined experimental temperature and position parameters, the experimental structure factors were scaled, in a least-squares sense, to the refined theoretical structure factors. This resulted in a new experimental scale factor. Next, a multipole refinement of the experimental data was performed, with the new scale factor kept fixed, and the process of matching and scaling, described above, was repeated

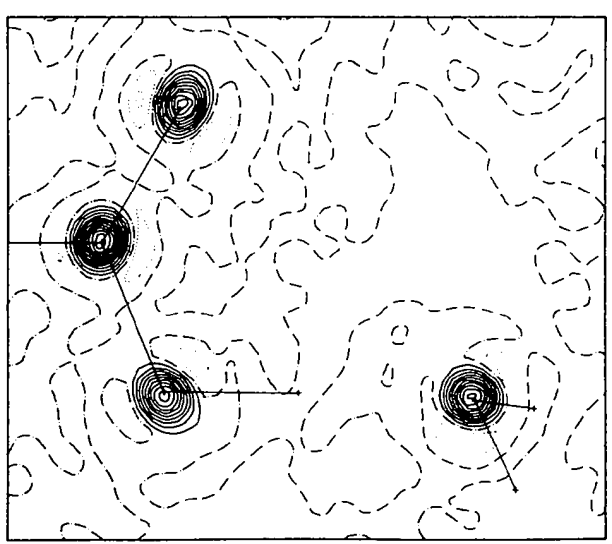

(a)

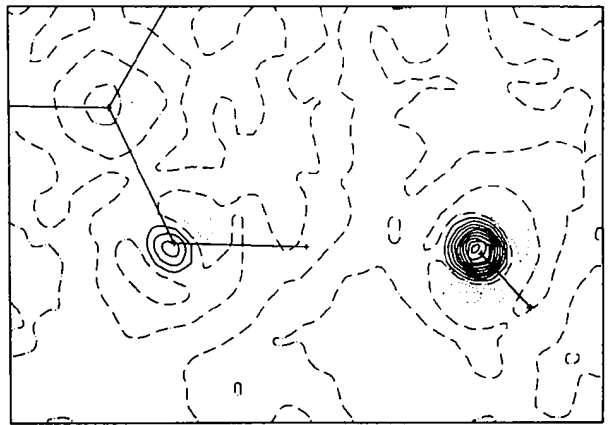

(b)

Fig. 3. Difference between the theoretical dynamic deformation density in oxalic acid dihydrate corresponding to the refined parameters and that corresponding to the input parameters, as specified in Table 2. The contour interval is $0.05 \mathrm{e}^{-3}$. (a) Difference in the plane of the oxalic acid molecule. (b) Difference in the plane perpendicular to the water molecule and bisecting the $\mathrm{H}(2)-\mathrm{O}(3)-\mathrm{H}(3)$ angle. 
until self-consistency had been reached. The experimental scale factor, obtained this way, is believed to be in close agreement with the true scale factor. With this scale factor kept fixed, a multipole refinement of the experimental data was performed, the positional and thermal parameters of which are summarized in Table 1 (refinement MR2). The corresponding experimental dynamic deformation density is depicted in Figs. 4(a), (b). These results justify a strong preference for the use of a scale factor obtained from a high-order refinement of the experimental data, which is kept fixed during the multipole refinement, instead of refining the scale factor during the multipole refinement.

Theory and experiment can now be compared. To do so, two different ways suggest themselves. With the experimental density distribution as reference, comparison of the distributions in real space is the obvious choice, whereas the quality of the experimental data, defined by the correspondence with theory, can better be judged in reciprocal space.

The difference of the refined experimental and refined theoretical dynamic density, obtained from matching

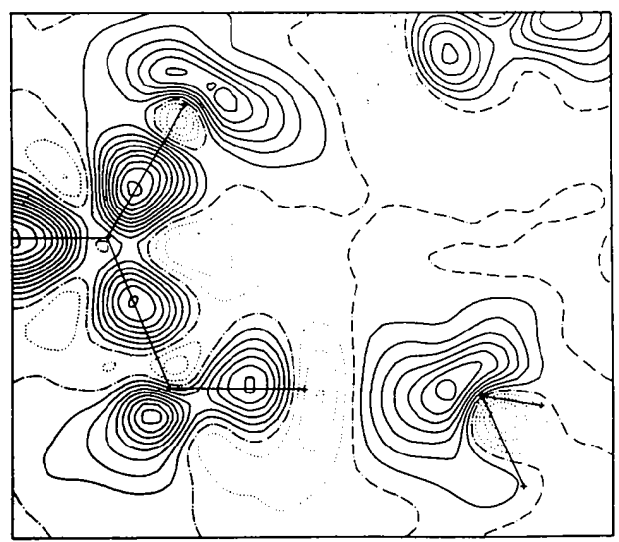

(a)

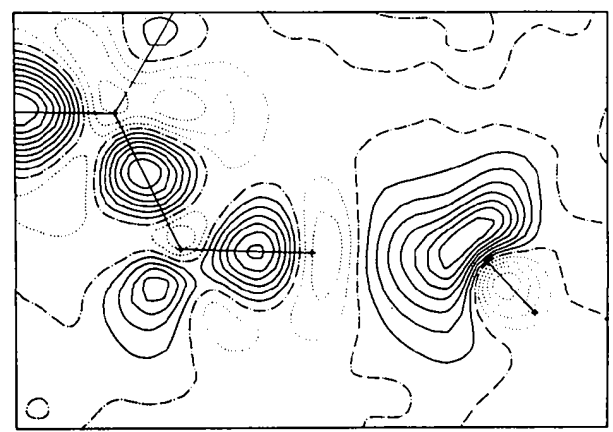

(b)

Fig. 4. Refined experimental dynamic deformation density in oxalic acid dihydrate, corresponding to a scale factor derived from a comparison with theoretical results. The contour interval is $0.05 \mathrm{e} \AA^{-3}$. (a) Deformation density in the plane of the oxalic acid molecule. (b) Deformation density in the plane perpendicular to the water molecule and bisecting the $\mathrm{H}(2)-\mathrm{O}(3)-\mathrm{H}(3)$ angle. refined experimental and refined theoretical position and thermal parameters together with a theoretically derived scale factor, as described above, is depicted in Figs. 5(a), $(b)$. The agreement between experiment and theory is seen to be remarkable. Differences between experiment and theory near the $\mathrm{C}$ and $\mathrm{O}$ atoms are not significant because of the large standard deviation at these positions. The discrepancy index between experiment and theory amounts to $w R=0.0106$.

With the aim to investigate the relative influence of the crystal environment on the electron-density distribution of the oxalic acid dihydrate complex, the density rearrangement upon placing the complex in the electric field, produced by the crystal environment, was calculated (Krijn \& Feil, 1988). The difference between the refined experimental and refined theoretical dynamic density, including the crystal-field effect, is depicted in Figs. $6(a),(b)$. None of the features in the density redistribution induced by the crystal environment is significant on its own. However, inclusion of the environment systematically improves the agreement between experiment and theory, as is reflected in a lowering of the $w R$ factor $(w R=0.0096)$.

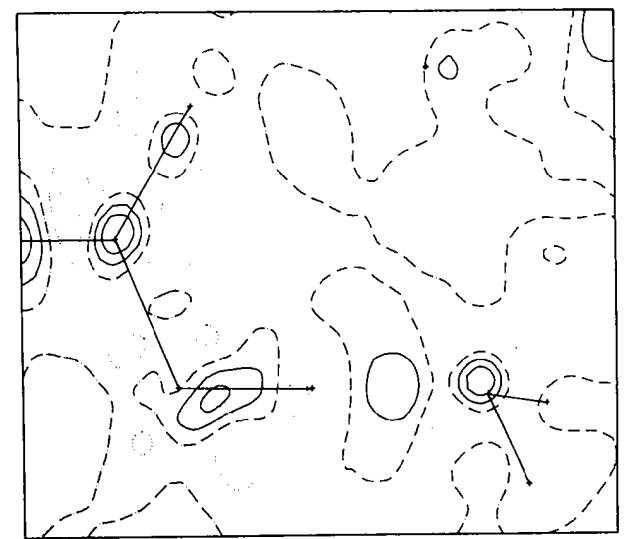

(a)

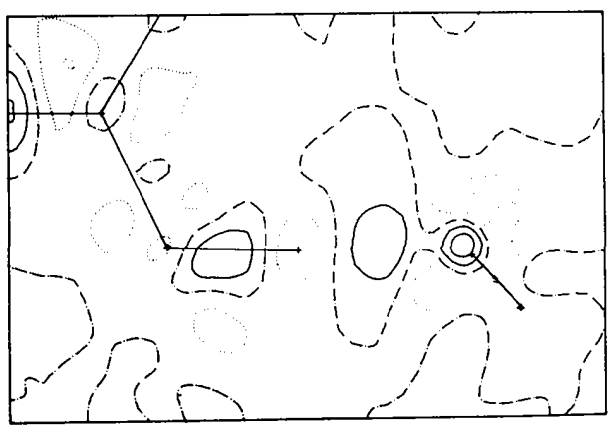

(b)

Fig. 5. Refined experimental dynamic density minus refined theoretical dynamic density in oxalic acid dihydrate. The contour interval is $0.05 \mathrm{e} \AA^{-3}$. (a) Difference in the plane of the oxalic acid molecule. $(b)$ Difference in the plane perpendicular to the water molecule and bisecting the $\mathrm{H}(2)-\mathrm{O}(3)-\mathrm{H}(3)$ angle. 
The results of a similar comparison of experiment and theory, without including hydrogen bonding and crystal environment in the theoretical results, is shown in Figs. $7(a),(b)$. Neglect of intermolecular interactions thus clearly deteriorates the agreement $(w R=0.0129$ ).

Assuming that no correlation between the errors in the refined experimental and refined theoretical density exists, we infer that the standard deviation in the dynamic experimental density is $0.05 \mathrm{e} \AA^{-3}$. Based on a comparison of deformation densities, Stevens (1980) arrived at a much larger deviation of $0.40 \mathrm{e} \AA^{-3}$, however, not necessarily inconsistent with the present results. Our conclusions are based on refined dynamic densities, whereas Stevens (1980) compares unrefined dynamic deformation densities. None of the features in the difference between experiment and theory (Figs. $5 a, b)$ seems to be significant. Discrepancies occur at sites where errors in the experimental density are known to accumulate (near atomic nuclei and centres of symmetry).

It was the asymmetry in the lone-pair deformation density distribution of the acceptor $\mathrm{O}$ atom, observed

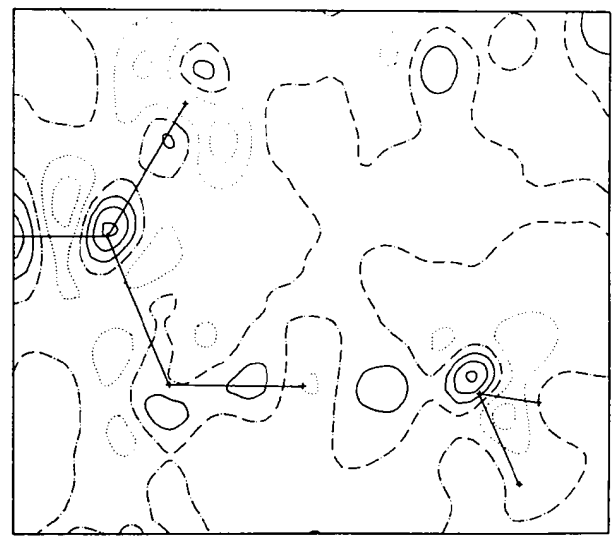

(a)

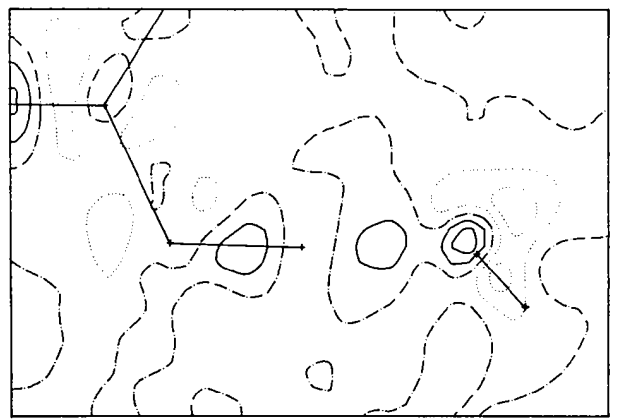

(b)

Fig. 6. Refined experimental dynamic density minus refined theoretical dynamic density in oxalic acid dihydrate, including crystal-field effects. The contour interval is $0.05 \mathrm{e}^{-3}$. (a) Difference in the plane of the oxalic acid molecule. $(b)$ Difference in the plane perpendicular to the water molecule and bisecting the $\mathrm{H}(2)-\mathrm{O}(3)-\mathrm{H}(3)$ angle. by Stevens \& Coppens (1980) and Dam et al. (1983) that suggested that the effect of hydrogen bonding could be observed by X-ray diffraction. Olovsson (1980) and Eisenstein \& Hirshfeld (1983) indicated recently that the superposition of the density distributions of the molecules constituting a hydrogen-bonded complex already results in an asymmetric lone-pair deformation density of the acceptor atom, since the donor density extends into the acceptor-atom lone-pair region. This superposition effect partially explained the results of his calculations in which the lone pair involved in hydrogen bonding was found to be weaker than the corresponding non-bonded one. The calculations of Breitenstein et al. (1983) on oxalic acid dihydrate showed a similar pattern. From both our experiments and calculations we infer, however, the opposite to be true. The lone-pair deformation density in the bonding region is the stronger one. It is the unexpected accuracy of the experimental density distribution that makes the density distribution upon hydrogen bonding observable. However, since experiment generally cannot provide

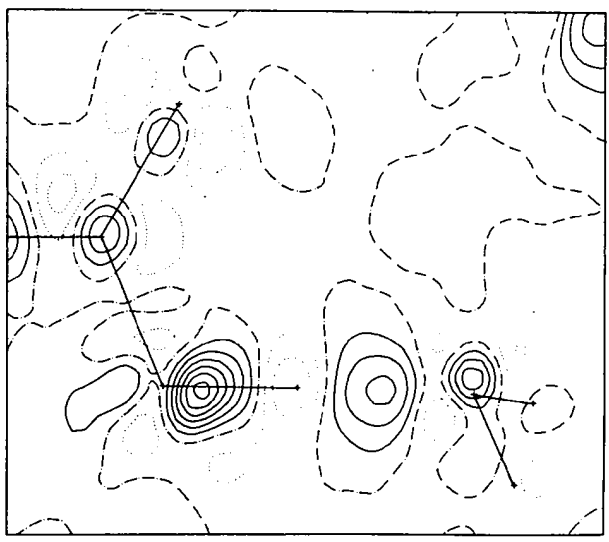

(a)

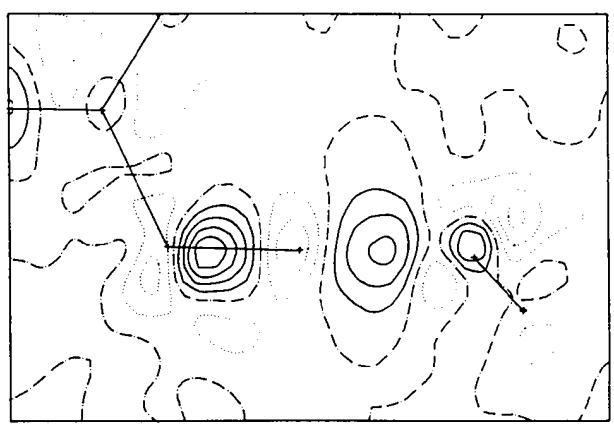

(b)

Fig. 7. Refined experimental dynamic density minus refined theoretical dynamic density of free oxalic acid and water in the geometry of the complex (i.e. neglecting all intermolecular and crystal-field effects). The contour interval is $0.05 \mathrm{e}^{-3}$. (a) Difference in the plane of the oxalic acid molecule. $(b)$ Difference in the plane perpendicular to the water molecule and bisecting the $\mathrm{H}(2)-\mathrm{O}(3)-\mathrm{H}(3)$ angle. 
the densities of the free molecules constituting the hydrogen-bonded complex, the deduction of density redistributions, consequent on hydrogen bonding, solely from the experiment is not straightforward.

Remaining discrepancies between theory and experiment may well be the result of anharmonicity in the thermal motion and an unphysical and unconstrained division of electron density among the atoms constituting the molecules in the crystal.

We now proceed to the comparison in reciprocal space. The structure factors were grouped in $(\sin \theta) / \lambda$ intervals of $0.10 \AA^{-1}$. For each interval, a discrepancy index between experiment and theory, $w R$, was calculated for each of the theoretical models used. The resulting curves were smoothed and are shown in Fig. 8. Results are seen to confirm fully the conclusions drawn above. The variation in $w R$ with $(\sin \theta) / \lambda$ is consistent with the spatial extent of the features in Figs. $5-7$.

\section{Concluding remarks}

A multipole-refinement-based comparison of the experimental and theoretical electron-density distributions results in a cancelation of refinement-model errors and elimination of statistical errors in the experimental density distribution. The experimental scale factor corresponds closely to the scale factor resulting from a high-order refinement of the experimental data instead of a multipole refinement.

The agreement between experimental and theoretical density distribution in $\alpha$-oxalic acid dihydrate is

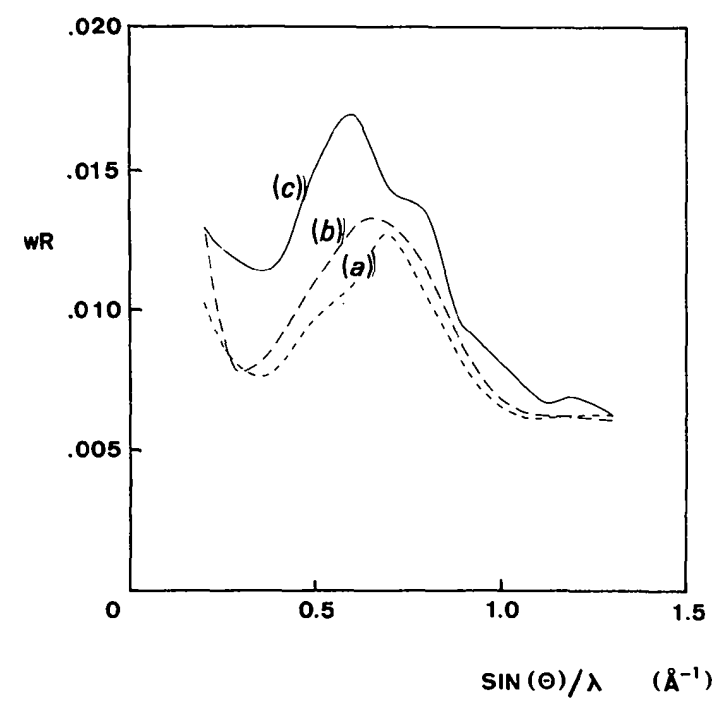

Fig. 8. Discrepancy index between experiment and theory, $w R$, as a function of $(\sin \theta) / \lambda$. (a) Including intermolecular and crystal-field effects in the theoretical calculation. (b) Including intermolecular and neglecting crystal-field effects in the theoretical calculation. (c) Neglecting both intermolecular and crystal-field effects in the theoretical calculation. excellent. Neglect of density redistributions in the theoretical density induced by hydrogen bonding markedly deteriorates the agreement. Inclusion of the effect of the crystal environment has only a minor influence.

The excellent agreement between experiment and theory confirms the usefulness of performing quantummechanical calculations, with a local approximation to exchange and correlation, as an alternative to the $\mathrm{HF}$ approach.

\section{References}

BaERends, E. J. \& Ros, P. (1978). Int. J. Quant. Chem. 12S, 169-190.

Baerends, E. J., Vernoous, P., RozendaAl, A., Boerrigter, P. M., KRIJN, M. P. C. M., FeIL, D. \& SundHOLM, D. (1985). J. Mol. Struct. 133, 147-159.

Bicerano, J., Marynick, D. S. \& Lipscomb, W. N. (1978). J. Am. Chem. Soc. 100, 732-739.

Boys, S. F. \& BERNARd, F. (1970). Mol. Phys. 19, 553-566.

Breitenstein, M., DanNöHl, H., Meyer, H., Schweig, A., Seeger, R., Seeger, U. \& Zittlau, W. (1983). Int. Rev. Phys. Chem. 3, 335-391.

Ceperley, D. M. \& Alder, D. J. (1980). Phys. Rev. Lett. 45, $566-569$.

Coppens, P., Dam, J., Harkema, S., Feil, D., Feld, R., Lehman, M. S., Goddard, R., Krüger, C., Hellner, E., JohanSEN, H., larsen, F. K., Koetzle, T. F., McMullan, R. K., Maslen, E. N. \& Stevens, E. D. (1984). Acta Cryst. A40, 184-195.

Coulson, C. A. \& Thomas, M. W. (1971). Acta Cryst. B27, 1354-1359.

Dam, J., Harkema, S. \& Fell, D. (1983). Acta Cryst. B39, 760-768.

Eisenstein, M. \& HiRshfeld, F. L. (1983). Acta Cryst. B39, 61-75.

Hamilton, W. C. (1964). Statistics in Physical Science, p. 34. New York: Ronald.

Hermansson, K. (1984). PhD Dissertation, Univ. of Uppsala, Sweden.

Hermansson, K. (1985). Acta Cryst. B41, 161-169.

HiRSHFELD, F. L. (1971). Acta Cryst. B27, 769-781.

HIRSHFELD, F. L. (1977). Isr. J. Chem. 16, 226-229.

Kaisser, P. \& SMITH, V. H. (1977). Adv. Quant. Chem. 10, 37-76.

Koetzle, T. F. \& McMullan, R. K. (1980). Data set N4 (Coppens et al., 1984).

Kohn, W. \& Sham, L. J. (1965). Phys. Rev. 140, A1133-A1138.

KRIN, M. P. C. M. \& FeIL, D. (1986). J. Chem. Phys. 85, 319-323.

Krin, M. P. C. M. \& FeIL, D. (1987). J. Phys. Chem. 91, 540-544.

KriJn, M. P. C. M. \& FeIL, D. (1988). J. Chem. Phys. In the press.

Olovsson, I. (1980). Electron and Magnetization Densities in Molecules and Crystals, edited by P. BECKER, pp. 831-894. New York: Plenum.

Stevens, E. D. (1980). Acta Cryst. B36, 1876-1886.

Stevens, E. D. \& Coppens, P. (1980). Acta Cryst. B36, 1864-1876.

StewART, R. F. (1976). Acta Cryst. A32, 565-574.

Stoll, H., GolkA, E. \& Preuss, H. (1980). Theor. Chim. Acta, 55, $29-41$.

Stoll, H., Pavlidou, C. M. E. \& Preuss, H. (1978). Theor. Chim. Acta, 49, 143-149.

Swaminathan, S., Craven, B. M., Spackman, M. A. \& Stewart, R. F. (1984). Acta Cryst. B40, 398-404.

Vosko, S. H. \& WILK, L. (1980). Phys. Rev. B, 22, 3812-3815.

Vosko, S. H., WILk, L. \& Nusair, M. (1980). Can. J. Phys. 58, $1200-1211$. 